\title{
Interaction of quantum systems with environment in QCD
}

\author{
Viatcheslav Kuvshinov ${ }^{1, *}$, Valery Shaparau ${ }^{1 * *}$, and Eugene Bagashov $^{1 * * *}$ \\ ${ }^{1}$ Joint Institute for Power and Nuclear Research - Sosny \\ National Academy of Science of Belarus \\ PO box 119, 220109 Minsk, Belarus
}

\begin{abstract}
It is shown that the interaction of quark with the stochastic vacuum of QCD (considered as an environment) leads to the decoherence of quark colour state, associated with the loss of information on the initial quark colour. We propose to consider this process as a reason of the confinement of the quark colour. Asymptotically this leads to confined quarks (fully mixed colourless quark states) in the limit of large distances and time intervals (confinement region) and free coloured quarks in the limit of small distances and time intervals (asymptotic freedom).

We propose quantitative characteristics that allow to describe the process of interaction: purity, fidelity, von Neumann entropy, quantum information measure. The cases of two and arbitrary number of quarks are considered, and it is shown that the entanglement in such system disappears in the limit of large distances and time intervals. The process is in good agreement with the known theorems in quantum information theory (no-cloning and no-hiding).

We study non-perturbative evolution of the gluon colour states during short time. Fluctuations of gluons are less than those for coherent states. This fact suggests that there gluon squeezed states can arise. Theoretical justification for the occurrence both singe- and two-mode gluon squeezing effects in QCD is given.

We show that gluon entangled states which are closely related with two-mode squeezed states of gluon fields can appear at short time non-perturbative evolution by analogy with corresponding photon states in quantum optics.
\end{abstract}

\section{Introduction}

Interactions of some quantum system with the environment can be represented by additional stochastic terms in the Hamiltonian of the system. The density matrix of the system in this case is obtained by averaging with respect to stochastic terms - degrees of freedom of the environment. Interactions with the environment result in decoherence of quantum superpositions $[1,2]$.

The model of QCD stochastic vacuum is one of the phenomenological models which explains quark confinement (Wilson loop decrease), string tensions and field configurations around static charges [3,4]. It is based on the assumption that one can calculate vacuum

\footnotetext{
*e-mail: kuvshinov2003@gmail.com

**e-mail: shaparau@sosny.bas-net.by

***e-mail: bagashov@sosny.bas-net.by
} 
expectation values of gauge invariant quantities as expectation values with respect to some well-behaved stochastic gauge field, for which only the second order correlators are important and the others are negligible [4].

Here we consider QCD stochastic vacuum as the environment for colour quantum particles and show that the result of their interaction is decoherence of quantum superpositions, loss of information and confinement of colour states phenomenon in the limit of large distances and time intervals [5-8].

Analogies between multiple hadron in high energy physics (HEP) and photon production in quantum optics (QO) discussed in [9-12]. It was shown, in particular, that the general distribution that characterizes $e^{+} e^{-}, p \bar{p}$, neutrino-induced collisions is a $k$-mode squeezed state distribution $[13,14]$, the multiplicity distribution of pions has been explained by the formalism of the squeezed isospin states [15].

Squeezed states (SS) provoke great interest in connection with their uncommon properties: sub-Poissonian (for coincide phases) or super-Poissonian (for antiphases) statistics corresponding to antibunching or bunching of photons [16-19]. The squeezed light is generated from the coherent one by nonlinear devices and is pure quantum non-perturbative phenomenon [17-19]. Moreover two-mode photon SS [18] are one of the examples of the entangled states [20] with their uncommon properties: if one photon is registered with defined polarization, the other photon immediately becomes opposite polarized. Thus a measurement over one particle has an instantaneous effect on the other, possibly located at a large distance.

Studying non-perturbative evolution of gluon states prepared by perturbative cascade stage in jets [21] we have proved within QCD that this stage of jet evolution can be a source of gluon SS by analogy with nonlinear devices in QO for photon [22-24]. In these papers we investigated single-mode squeezing effect for virtual gluons with definite colour and vector components during small temporal evolution.

Developing the analogy with QO it can be shown that the two-mode (in particular, collinear) gluon squeezed states can be entangled ones [25]. Such gluon states can lead to $q \bar{q}$ entangled ones. Interaction of the quark entangled states with stochastic vacuum (quantum measurement) has a remarkable property, namely, as soon as some measurement projects one quark onto a state with definite colour, the another quark also immediately obtains opposite colour that leads to coupling of quark-antiquark pair, string tension inside $q \bar{q}$-pair and free propagation of colourless hadrons.

\section{Evolution of single-particle colour states and colour superpositions}

The colour state of a spinless heavy quark moving along the trajectory $\gamma$ is described by the parallel transport operator $[4,26]$ :

$$
|\phi(\gamma)\rangle=\mathcal{P} \exp \left(i \int_{\gamma} d x^{\mu} \hat{A}_{\mu}\right)\left|\phi_{\text {in }}\right\rangle,
$$

where $\mathcal{P}$ is the path-ordering operator, and $\hat{A}_{\mu}$ is the gauge field. In order to make this expression gauge invariant, we also have to demand that the path $\gamma$ should be a closed loop, which physically corresponds to the situation when a particle-antiparticle pair is created, propagates and annihilates [3, 4].

Consider QCD stochastic vacuum as environment. Let $\rho(\gamma)$ be the density matrix, describing the state of quark under the current environmental conditions. After the integration 
over the implementations of the stochastic vacuum we get the following expression of density matrix of quark $[5,8]$ :

$$
\rho(\gamma)=N_{c}^{-1}+\left(\left|\phi_{i n}\right\rangle\left\langle\phi_{i n}\right|-N_{c}^{-1}\right) W_{a d j}(\gamma),
$$

where $W_{a d j}(\gamma)$ is the Wilson loop in the adjoint representation. By definition, in fundamental representation the Wilson loop is written as [4]

$$
W_{\text {fund }}(\gamma)=\operatorname{Tr}\left(\mathcal{P} \exp \left(i \int_{\gamma} d x^{\mu} \hat{A}_{\mu}\right)\right) .
$$

Exponential decay of Wilson loop is a well recognized criterion of confinement $[3,4]$.

Consider for simplicity a case of a rectangular loop spanning the time $T$ and distance $R$. In this case the expression (2) might be written as

$$
\rho(\gamma)=N_{c}^{-1}+\left(\rho_{i n}-N_{c}^{-1}\right) \exp \left(-\sigma_{a d j} R T\right)
$$

where $\sigma_{a d j}$ is the QCD string tension in the adjoint representation.

In case of a system consisting of a quark in a state of arbitrary colour and its environment (QCD stochastic vacuum) the interaction between the quark and the vacuum in the limit of large $R T$ leads to the emergence of a mixed state with equal probabilities for all the colours and disappearance of the non-diagonal terms in the density matrix. Effectively, the interaction leads to the transition of density matrix $\rho_{\text {in }} \rightarrow \operatorname{diag}\left(N_{c}^{-1}\right)$ [27].

It is also possible to consider the case close to the asymptotics $R T \rightarrow 0$, that is, in a perturbative area. Here we would have $0<\sigma_{a d j} R T \ll 1$ and then the density matrix expression would change to

$$
\rho(\gamma)=\rho_{\text {in }}-\left(\rho_{\text {in }}-N_{c}^{-1}\right) \sigma_{\text {adj }} R T .
$$

\section{Quantitative characteristics of interaction}

Decoherence rate during the interaction of quark with stochastic vacuum might be evaluated basing on purity $P$ of its quantum state [2]:

$$
P=\operatorname{Tr}\left(\rho^{2}\right)
$$

In our case, we get

$$
P=N_{c}^{-1}+\left(1-N_{c}^{-1}\right) W_{a d j}^{2}(\gamma) .
$$

Obviously, when $R T \rightarrow 0$ and thus $W_{a d j}(\gamma) \rightarrow 1$, we get $P=1$, which corresponds to a pure colour state. And in the case of $R T \rightarrow \infty$ and thus $W_{a d j}(\gamma) \rightarrow 0$ we get $P=N_{c}^{-1}$, which is the minimal possible value, corresponding to a fully mixed colourless state, i.e. an equal probability for all the colours.

Other useful parameter for the quantitative description of the interaction of quark with stochastic vacuum is von Neumann entropy [28]:

$$
S=-\operatorname{Tr}(\rho \ln \rho)
$$

Here $S=0$ for the initial state (since $\rho_{\text {in }}^{2}=\rho_{\text {in }}$ ) and $S=\ln N_{c}$ in case of $R T \rightarrow \infty$. So we might conclude that the information (that might be written as $I=1-\frac{S}{\ln N_{c}}$ ) on the initial quark colour state is lost because of its interaction with the stochastic vacuum, which causes the decoherence of its quantum state. 
Another parameter that we might use is quantum fidelity $[5,8]$ :

$$
f=\left\langle\left(\left\langle\phi_{\text {in }}\left|\mathcal{P} \exp \left(\int i \hat{A_{\mu}} d x^{\mu}\right)\right| \phi_{i n}\right\rangle\right)\right\rangle .
$$

The final expression for the fidelity of the particle moving in the Gaussian-dominated stochastic vacuum is

$$
f=\exp \left(-\frac{1}{2} g^{2} l_{c o r r}^{2} F^{2} R T\right),
$$

where $g$ is coupling constant, $l_{\text {corr }}$ - correlation length in the QCD stochastic vacuum, $F^{2}-$ average of the second cumulant of curvature tensor.

The fidelity in this case decays exponentially with time. This hints on a close connection between confinement and instability of colour particle motion.

\section{Interaction of multiparticle states with QCD stochastic vacuum}

\subsection{Two particles}

Consider a system of two quarks, denoted as $A$ and $B$. Also consider that there is only two possible colour states for each of them: $|A\rangle,|B\rangle$ and $|\bar{A}\rangle,|\bar{B}\rangle$. Thus $N_{c}=2$.

Here is the list of possible types of states, in which the system may be found (excluding some specific types e.g. as the squeezed states):

- By purity: pure, mixed;

- By separability: separable, non-separable (entangled).

Pure separable system state vector of two quarks can be presented as

$$
|\phi\rangle=|a\rangle|b\rangle,
$$

where $|a\rangle$ and $|b\rangle$ are some states of subsystems $A$ and $B$. Here we may consider the evolution of each quark independently, assuming $N_{c}=2$.

Mixed separable state gives us similar picture, with the only difference being the absence of a state vector. The density matrix of the system will have the form

$$
\rho_{A B}=\rho_{A} \rho_{B},
$$

where density matrices $\rho_{A}$ and $\rho_{B}$ correspond to states of $A$ and $B$ quarks. $\rho_{A}$ and $\rho_{B}$ therefore may also be considered independently with the help of the described mechanism. We will choose pure non-separable state in the form

$$
|\phi\rangle=\alpha|A\rangle|\bar{B}\rangle+\beta|\bar{A}\rangle|B\rangle .
$$

Density matrix in the basis of the corresponding vectors for such a state will have the form

$$
\begin{aligned}
& \langle B \bar{A}|\langle\bar{B} A| \\
& \rho_{A B}=|\phi\rangle\langle\phi|={ }_{|A \bar{B}\rangle}^{|\bar{A} B\rangle}\left(\begin{array}{cc}
|\beta|^{2} & \alpha^{*} \beta \\
\alpha \beta^{*} & |\alpha|^{2}
\end{array}\right)
\end{aligned}
$$

During the evolution and the interaction with vacuum under the condition $R T \rightarrow \infty$ this state will take the diagonal form, also keeping in mind that it should be raised to a power of $N_{p}=2$, where $N_{p}$ is the number of particles. In a given basis (under the condition $W(\gamma) \rightarrow 0$ ) the resulting density matrix can be represented as a diagonal: $\rho_{A B_{W \rightarrow 0}}=\frac{1}{4}$.

It can be seen that the entanglement present in (13) vanishes, since the resulting density matrix can be presented in the form $\sum_{i} k_{i} \rho_{i 1} \rho_{i 2}$, where $k_{i}$ is some weight factor, $\rho_{i 1}$ and $\rho_{i 2}$ some density matrices. 


\subsection{Arbitrary number of particles}

In case of $N_{p}$ quarks in the system Wilson loop contour should be encircled $N_{p}$ times, and each time we should perform the appropriate functional integration (or, as the stochastic vacuum is homogeneous [3], we might just take the $N_{p}$ power of the Wilson loop in the first place). So the expression for the density matrix of the system after the interaction with the vacuum (2) becomes [29]:

$$
\rho(\gamma)=N_{c}^{-N_{p}}+\left(\rho_{\text {in }}-N_{c}^{-N_{p}}\right) W_{a d j}^{N_{p}}
$$

As before, it is possible to calculate accordingly the characteristics of quantum purity and entropy for different cases (namely, before and after the interaction).

\section{The gluon state non-perturbative evolution in QCD during a short time}

Calculations performed within perturbative QCD [32, 33] show that multiplicity distribution (MD) at the end of the perturbative cascade is close to a negative binomial distribution. Moreover gluon MD in the range of the small transverse momenta is Poissonian [21]. Thus gluon MD at the end of the perturbative cascade can be represented as a combination of Poissonian distributions each of which corresponds to a coherent state. So we can suggest that set of the gluon coherent states with different colours and vector components describes the gluon system at end of perturbative stage and then the initial state vector $\mid$ in $\rangle$ for investigation of the non-perturbative evolution is

$$
\mid \text { in }\rangle \equiv|\alpha\rangle=\prod_{c=1}^{8} \prod_{l=1}^{3}\left|\alpha_{l}^{c}\right\rangle,
$$

where $\left|\alpha_{l}^{c}\right\rangle$ is a gluon coherent state vector. The gluon state non-perturbative evolution during a short time $t$ is defined as

$$
|\mathrm{f}\rangle \simeq\left(1-\text { it } H_{\mathrm{I}}\right)|\mathrm{in}\rangle,
$$

where $H_{\mathrm{I}}=H_{\mathrm{I}}^{(3)}+H_{\mathrm{I}}^{(4)}$ is the Hamiltonian ${ }^{1}$ three-gluon $\left(H_{\mathrm{I}}^{(3)}\right)$ and four-gluon $\left(H_{\mathrm{I}}^{(4)}\right)$ selfinteractions

$$
\begin{aligned}
H_{\mathrm{I}}^{(3)} & =g \int f_{a b c} \mathbf{E}_{a} \mathbf{A}_{b} A_{c}^{0} d^{3} x-\frac{g}{2} \int f_{a b c} \mathbf{B}_{a}\left[\mathbf{A}_{b} \mathbf{A}_{c}\right] d^{3} x, \\
H_{\mathrm{I}}^{(4)} & =\frac{g^{2}}{2} \int\left(f_{a b c} \mathbf{A}_{b} A_{c}^{0}\right)^{2} d^{3} x+\frac{g^{2}}{8} \int\left(f_{a b c}\left[\mathbf{A}_{b} \mathbf{A}_{c}\right]\right)^{2} d^{3} x,
\end{aligned}
$$

which explicit forms in momentum representation include the squares of the creation and annihilation operators for gluons with specified colour and vector indices [25].

As is known from quantum mechanics and quantum optics, the presence of such structure in the Hamiltonian and, consequently, in the evolution operator is a necessary condition for emergence of squeezed states [16-19], since the unitary squeezing operator involves quadratic combinations of the creation and annihilation operators

$$
S(z)=\exp \left\{\frac{z^{*}}{2} a^{2}-\frac{z}{2}\left(a^{+}\right)^{2}\right\}
$$

where $z=r e^{i \vartheta}$ is an arbitrary complex number, $r$ is a squeeze factor, phase $\vartheta$ defines the direction of squeezing maximum.

\footnotetext{
${ }^{1}$ Here we consider the case when a motion equation is satisfied, i.e. $\partial_{\mu} F_{a}^{\mu v}+g f_{a b c} A_{\mu}^{b} F_{c}^{\mu v}=0$.
} 


\section{Gluon squeezed states production in QCD}

\subsection{Single-mode squeezing for gluons}

In order to verify whether the final gluon state vector $|\mathrm{f}\rangle$ (17) describes the single-mode SS, it is necessary to introduce the phase-sensitive Hermitian operators $\left(X_{l}^{h}\right)_{1}=\left[a_{l}^{h}+\left(a_{l}^{h}\right)^{+}\right] / 2$ and $\left(X_{l}^{h}\right)_{2}=\left[a_{l}^{h}-\left(a_{l}^{h}\right)^{+}\right] / 2 i$ by analogy with quantum optics $[16,18,19]$ and to establish conditions under which the variance of one of them can be less than the variance of a coherent state. Here $a_{l}^{h}\left(a_{l}^{h+}\right)$ are the operators annihilating (creating) of gluons with colours $h=\overline{1,8}$ and vector index $l=\overline{1,3}$.

Condition of the single-mode squeezing for gluon with fixed colour $h$ and vector component $l$ is expressed in the form of the inequalities [22-24]

$$
\left\langle N\left(\Delta\left(X_{l}^{h}\right)_{2}\right)^{2}\right\rangle<0
$$

Here $N$ is the normal-ordering operator such as

$$
\left\langle N\left(\Delta\left(X_{l}^{h}\right)_{2}\right)^{2}\right\rangle= \pm \frac{1}{4}\left\{\left[\left\langle\left(a_{l}^{h}\right)^{2}\right\rangle-\left\langle a_{l}^{h}\right\rangle^{2}\right]+\left[\left\langle\left(a_{l}^{h+}\right)^{2}\right\rangle-\left\langle a_{l}^{h+}\right\rangle^{2}\right] \pm 2\left[\left\langle a_{l}^{h+} a_{l}^{h}\right\rangle-\left\langle a_{l}^{h+}\right\rangle\left\langle a_{l}^{h}\right\rangle\right]\right\} \text {. }
$$

Performing averaging in expression (22) over the evolved vector $|\mathrm{f}\rangle$ (17) we obtain singlemode squeezing condition in the form

$$
\left\langle N\left(\Delta\left(X_{l}^{h}\right)_{\frac{1}{2}}\right)^{2}\right\rangle= \pm \frac{i t}{4}\left(\left\langle\alpha\left|\left[\left[H_{\mathrm{I}}, a_{l}^{h+}\right], a_{l}^{h+}\right]\right| \alpha\right\rangle-\langle h . c .\rangle\right)<0 .
$$

The three-gluon self-interaction does not lead to the single-mode squeezing of the gluons since

$$
\left[\left[H_{\mathrm{I}}^{(3)}, a_{l}^{h+}\right], a_{l}^{h+}\right]=0, \quad\left[a_{l}^{h},\left[a_{l}^{h}, H_{\mathrm{I}}^{(3)}\right]\right]=0 \quad \text { when } \quad f_{h h a}=0 .
$$

Thus only the four-gluon self-interaction can give the single-mode squeezing effect during short time of the non-perturbative evolution.

For visualization, let us investigate the obtained single-mode squeezing condition (23) for selected gluon with colour $h$ in case when other gluon fields are collinear with respect to given one. In this case corresponding squeezing condition is

$$
\left\langle N\left(\Delta\left(X_{l}^{h}\right)_{2}\right)^{2}\right\rangle= \pm \frac{t g^{2}}{8 k_{0}^{2}(2 \pi)^{3}} f_{\text {hab }} f_{\text {hac }} \sum_{j, m \neq l}\left[\left(1+\frac{k_{l}^{2}}{k_{0}^{2}}\right) \delta_{j m}+\frac{k_{j} k_{m}}{k_{0}^{2}}\right]\left|\alpha_{j}^{b} \| \alpha_{m}^{c}\right| \sin \left(\gamma_{j}^{b}+\gamma_{m}^{c}\right)<0 .
$$

Here we have taken into account that $\alpha_{j}^{b}=\left|\alpha_{j}^{b}\right| e^{i \gamma_{j}^{b}}$ and $\alpha_{m}^{c}=\left|\alpha_{m}^{c}\right| e^{i \gamma_{m}^{c}}$. The single-mode squeezing condition is fulfilled for any cases apart from $\gamma_{j}^{b}+\gamma_{m}^{c}=0, \pi$ or if all initial gluon coherent fields are real or imaginary.

Obviously, single-mode squeezing effect for the gluon field with fixed colour and vector component is defined by the gluon coherent fields with other colours and vector indexes different from the selected one. Moreover the larger are both amplitudes of such gluon coherent fields, the larger is the squeezing effect. 


\subsection{Gluon two-mode colour squeezed states}

In order to verify whether the gluon state vector describes the two-mode SS with colours $h$ and $g$, it is necessary to introduce the phase-sensitive Hermitian operators $\left(X_{l}^{h, g}\right)_{1}=\left[a_{l}^{h}+a_{l}^{g}+a_{l}^{h+}+a_{l}^{g+}\right] /(2 \sqrt{2})$ and $\left(X_{l}^{h, g}\right)_{2}=\left[a_{l}^{h}+a_{l}^{g}-a_{l}^{h+}-a_{l}^{g+}\right] /(2 i \sqrt{2})$ by analogy with quantum optics $[18,19]$ and to establish conditions under which the variance of one of them can be less than the variance of a coherent state.

Condition of the two-mode colour squeezing is expressed in the form of the inequality [25]

$$
\left\langle N\left(\Delta\left(X_{l}^{h, g}\right)_{2}\right)^{2}\right\rangle<0
$$

Here $N$ is the normal-ordering operator.

So the two-mode colour squeezing condition is written in the form

$$
\begin{aligned}
\left\langle N\left(\Delta\left(X_{l}^{h, g}\right)_{\frac{1}{2}}\right)^{2}\right\rangle= & \frac{1}{2}\left(\left\langle N\left(\Delta\left(X_{l}^{h}\right)_{2}\right)^{2}\right\rangle+\left\langle N\left(\Delta\left(X_{l}^{g}\right)_{\frac{1}{2}}\right)^{2}\right\rangle\right) \\
& \pm \frac{i t}{4}\left(\left\langle\alpha\left|\left[\left[H_{\mathrm{I}}, a_{l}^{h+}\right], a_{l}^{g+}\right]\right| \alpha\right\rangle-\langle\text { h.c. }\rangle\right)<0 .
\end{aligned}
$$

In this case as in the previous one the three-gluon self-interaction also does not lead to the two-mode colour squeezing effect by virtue of (24) and

$$
\left[\left[H_{\mathrm{I}}^{(3)}, a_{l}^{h+}\right], a_{l}^{g+}\right]=0, \quad\left[a_{l}^{g},\left[a_{l}^{h}, H_{\mathrm{I}}^{(3)}\right]\right]=0 .
$$

Thus only the four-gluon self-interaction can lead to the squeezing effect during a short non-perturbative time evolution.

For visualization, let us investigate the obtained two-mode squeezing condition (27) for selected gluons with colours $h$ and $g$ with equal momenta and vector components in case when other gluon fields are collinear with respect to the given ones. In this case corresponding squeezing condition is

$$
\begin{aligned}
\left\langle N\left(\Delta\left(X_{l}^{h, g}\right)_{2}\right)^{2}\right\rangle= & \pm \frac{t g^{2}}{16 k_{0}^{2}(2 \pi)^{3}}\left(f_{h a b} f_{h a c}+f_{g a b} f_{g a c}+2 f_{h a b} f_{g a c}\right) \\
& \times \sum_{j, m \neq l}\left[\left(1+\frac{k_{l}^{2}}{k_{0}^{2}}\right) \delta_{j m}+\frac{k_{j} k_{m}}{k_{0}^{2}}\right]\left|\alpha_{j}^{b} \| \alpha_{m}^{c}\right| \sin \left(\gamma_{j}^{b}+\gamma_{m}^{c}\right)<0 .
\end{aligned}
$$

The two-mode squeezing condition is fulfilled under the same conditions as in the singlemode one. Obviously, conclusions made for single-mode squeezing are valid in the case of the two-mode squeezing.

Thus non-perturbative gluon evolution is very significant under investigation of the squeezing effect.

\section{Entangled collinear gluon states}

A entangled state at finite squeezing parameter $r$ is known from quantum optics as a twomode squeezed state $[18,20]$

$$
|\mathrm{f}\rangle=S_{12}(r)|0\rangle_{1}|0\rangle_{2}=\frac{1}{\cosh r} \sum_{n=0}^{\infty}(\tanh r)^{n}|n\rangle_{1}|n\rangle_{2},
$$


where $S_{12}(r)=\exp \left\{r\left(a_{1}^{+} a_{2}^{+}-a_{1} a_{2}\right)\right\}$ is operator of two-mode squeezing. It is not difficult to demonstrate that the state vector $|\mathrm{f}\rangle$ describes the entangled state. Each of these entangled states has a uncommon property: a measurement over one particle have an instantaneous effect on the other, possibly located at a large distance.

The dimensionless coefficient [34]

$$
y=\left[\frac{\left|\overline{a_{1} a_{2}^{+}}\right|^{2}+\left|\overline{a_{1} \hat{a}_{2}}\right|^{2}}{2\left(\overline{a_{1}^{+} a_{1}}+1 / 2\right)\left(\overline{a_{2}^{+} a_{2}}+1 / 2\right)}\right]^{1 / 2}
$$

is the measure of entanglement for two-mode states, $0 \leq y<1$ (entanglement is not observe when $y=0)$. Here $\overline{a_{i} a_{j}^{+}}=\left\langle a_{i} a_{j}^{+}\right\rangle-\left\langle a_{i}\right\rangle\left\langle a_{j}^{+}\right\rangle, a_{i}, \hat{a}_{j}^{+}$are the annihilation and creation operators correspondingly.

Thus one of an entangled condition [34] is

$$
0<y<1 .
$$

By analogy with QO we assume that two-mode gluon SS with fixed colours $h, g$ is closely connected with corresponding entangled states of the gluons. Then the expression (31) for the entanglement coefficient in case two-mode gluon states [35] is

$$
y=\left[\frac{\left|\overline{a_{l}^{h} a_{l}^{g+}}\right|^{2}+\left|\overline{a_{l}^{h} a_{l}^{g}}\right|^{2}}{2\left(\overline{a_{l}^{h+} a_{l}^{h}}+1 / 2\right)\left(\overline{a_{l}^{g+} a_{l}^{g}}+1 / 2\right)}\right]^{1 / 2} .
$$

Averaging the annihilation and creation operators $a_{l}^{h}, a_{l}^{g}, a_{l}^{h+}, a_{l}^{g+}$ (33) over the evolved vector $|\mathrm{f}\rangle(17)$ we write the entanglement coefficient $y$ in the form

$$
y=t \sqrt{2\left\langle\alpha\left|\left[\left[H_{\mathrm{I}}, a_{l}^{h+}\right], a_{l}^{g+}\right]\right| \alpha\right\rangle\left\langle\alpha\left|\left[a_{l}^{g},\left[a_{l}^{h}, H_{\mathrm{I}}\right]\right]\right| \alpha\right\rangle} .
$$

Obviously, only the four-gluon self-interaction can yield entanglement effect during short time of the non-perturbative evolution according to (28).

For visualization, let us investigate the obtained entanglement coefficient (34) for example of the collinear gluons. In this case the entanglement condition (32) is written as

$$
0<\frac{t g^{2}}{2 \sqrt{2} k_{0}^{2}(2 \pi)^{3}}\left|f_{\text {hab }} f_{\text {gac }} \sum_{j, m \neq l}\left[\left(1+\frac{k_{l}^{2}}{k_{0}^{2}}\right) \delta_{j m}+\frac{k_{j} k_{m}}{k_{0}^{2}}\right] \alpha_{j}^{b} \alpha_{m}^{c}\right|<1 .
$$

Thus by analogy with quantum optics as a result of four-gluon self-interaction we obtain two-mode squeezed gluon states which are also entangled.

\section{Conclusions}

It has been shown that the interaction of quarks with the environment represented by QCD stochastic vacuum leads to the decoherence of the initial colour states of quarks and thus results in the loss of the information on their initial colour. Effectively in the limit of large distances and/or time intervals (confinement region) this leads to the inability to observe coloured objects, as they turn into colorless mixture with equal probabilities for each colour. Thus it is possible to treat the confinement of quarks in the framework of quantum information theory, considering it as a result of the decoherence of the colour state of quark due to its interaction with the stochastic QCD vacuum. 
It is worth noting that this process is in good agreement with the known statements in the quantum information theory itself: namely, the no-cloning [30] and no-hiding [31] theorems. The first one implies that it is impossible to measure a quantum state without disturbing it. Which means that measurement erases initial quantum information from the measured system. So in our case it is possible to say that the interaction of a quark with the stochastic vacuum of QCD leads to the loss of information on the colour state of quark itself. And one of the consequences of the second theorem is the fact that the quantum information is never lost entirely. During the measurement it redistributes to the environmental degrees of freedom. So in case of the interaction of a quark with the stochastic vacuum the information on the initial colour of the quark is being transferred to the stochastic vacuum.

Investigating of the gluon fluctuations at the non-perturbative stage we have proved theoretically the possibility of existence of both single- and two-mode gluon squeezed states. The emergence of such remarkable states becomes possible owing to the four-gluon selfinteraction. The three-gluon self-interaction does not lead to the squeezing effect during short time.

We have shown that QCD evolution during a short time leads both to colour squeezing and entanglement of gluons. It should be noted that the greater are both the amplitudes of the initial gluon coherent fields with different colour and vector indexes, the greater are squeezing and entanglement effects of the colour gluons.

Two-mode gluon states with two different colours can lead to $q \bar{q}$-entangled states role of which could be very significant for understanding of the hadronization and confinement phenomena.

\section{References}

[1] F. Haake, Quantum Signatures of Chaos (Springer-Verlag, Berlin, 1991)

[2] A. Peres, Quantum Theory: Concepts and Methods (Kluwer, Dordrecht, 1995)

[3] A. D. Giacomo, H. Dosch, V. I. Shevchenko, and Y. A. Simonov, Physics Reports 372, no. 4, 319-368 (2002)

[4] Y. A. Simonov, Uspekhi Fizicheskih Nauk. 166, 337-362 (1996) (in Russian)

[5] V. Kuvshinov, A. Kuzmin, Physics Letters A 316, 391-394 (2003)

[6] V. Kuvshinov, A. Kuzmin, Gauge Fields and Theory of Deterministic Chaos (Belorussian Science, Minsk, 2006, in Russian), 1-268

[7] V. Kuvshinov, P. Buividovich, Nonlinear Phenomena in Complex Systems 8, 313-316 (2005)

[8] V. Kuvshinov, P. Buividovich, Acta Physica Polonica B (Proceedings Supplement) 1(3), 579-582 (2008)

[9] A. Giovannini, Nuovo Cimento A 15, 543 (1973)

[10] W. Knox, Phys. Rew. D 10, 65 (1974)

[11] C. C. Shih, Phys. Rev. D 34, 2720 (1986).

[12] P. Carruthers and C. C. Shih, In. J. Mod. Phys. 2, 1447 (1987)

[13] B. A. Bambah and M. V. Satyanarayana, Phys. Rev. D 38, 2202 (1988)

[14] A. Vourdas and R. M. Weiner, Phys. Rev. D 38, 2209 (1988)

[15] I. M. Dremin and R. C. Hwa, Phys. Rev. D 53, 1216 (1996)

[16] S.Ya. Kilin, Quantum Optics (Minsk, 1990) (in Russian)

[17] O. Hirota, Squeezed light (Elsevier, Japan, Tokyo, 1992)

[18] D. F. Walls and G. J. Milburn, Quantum Optics (Springer-Verlag, NY., USA, 1995) 
[19] M. O. Scully and M.S. Zubairy, Quantum Optics (Cambridge University Press, Cambridge: 1997)

[20] S.Ya. Kilin, Quanta and Information. Chapter 1, Progress in Optics, Ed. by E. Wolf (2001, vol. 42), pp. 1-91

[21] S. Lupia, W. Ochs, J. Wosiek, Nucl. Phys. B 540, 405-433 (1999)

[22] V.I. Kuvshinov, V.A. Shaporov, Acta Phys. Pol. B 30(1), 59-68 (1999)

[23] V.I. Kuvshinov, V.A. Shaparau, Nonlinear Phenomena in Complex Systems 3(1), 28-36 (2000)

[24] V.I. Kuvshinov, V.A. Shaparau, Physics of Atomic Nuclei 65(2), 309-314 (2002)

[25] V.I. Kuvshinov, V.A. Shaparau, Nonlinear Phenomena in Complex Systems 6(4), 898$902(2003)$

[26] W. H. Zurek, in Proceedings of the Poincare Seminar 2005, Birkhauser (Basel, 2007)

[27] V. I. Kuvshinov and E. G. Bagashov, Theoretical and Mathematical Physics 184(3), 1304-1310 (2015)

[28] J. von Neumann, Mathematical Foundations of Quantum Mechanics (Princeton University Press, Princeton, 1955)

[29] V. Kuvshinov, E. Bagashov, Physics of Particles and Nuclei 48(5), 834-835 (2017)

[30] J. Park, Foundations of Physics 1, 23-33 (1970)

[31] S. L. Braunstein and A. K. Pati, Phys. Rev. Lett. 98, 080502 (2007)

[32] E.D. Malaza, B.R. Webber, Nucl. Phys. B 267, 702-713 (1986)

[33] I.M. Dremin, R.C. Hwa, Phys. Rev. D 49(11), 5805-5811 (1994)

[34] V.V. Dodonov, A.S.M. de Castro, S.S. Mizrahi, Phys. Lett. A 296(2\&3), 73-81 (2002)

[35] V.I. Kuvshinov, V.A. Shaparau, Nonlinear Phenomena in Complex Systems 17(4), 426429 (2014) 